\title{
Gesundheitskompetenz und die Corona-Krise
}

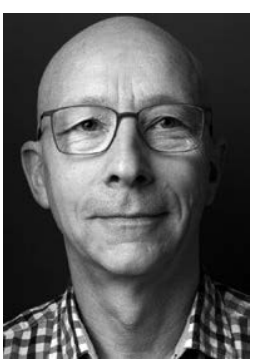

Thomas Abel

\author{
Bibliografie \\ DOI https://doi.org/10.1055/a-1099-8110 \\ Gesundheitswesen 2020; 82: 301-302 \\ (c) Georg Thieme Verlag KG Stuttgart · New York \\ ISSN 0941-3790 \\ Korrespondenzadresse \\ Prof. Dr. Thomas Abel \\ Institute of Social and Preventive Medicine \\ University of Bern \\ Mittelstrasse 43 \\ 3012 Bern (Schweiz) \\ thomas.abel@ispm.unibe.ch
}

„Wir sind eine Demokratie. Wir leben nicht von Zwang, sondern von geteiltem Wissen und Mitwirkung“

Angela Merkel, TV-Ansprache an die Nation, 18.3.2020

Wenn es um Infektionskrankheiten geht, so waren unsere Gesundheitssysteme immer schon auf das aktive Mittun der Bevölkerung angewiesen. Die Menschen mussten sich auch früher schon angemessen verhalten, z. B. mit Hygienemaßnahmen im Alltag, um Infektionsketten zu unterbrechen, um Versorgungssysteme nicht zu überlasten oder anderweitig mithelfen, Schlimmeres zu verhindern.

Und doch ist heute einiges anders: Nie zuvor gab es mehr frei verfügbare Informationen zu Gesundheit und Erkrankungsrisiko und nie zuvor war der Anspruch höher, dass Gesundheit machbar, Krankheit beherrschbar ist, wir alles im Griff haben. Diese immer stärker ausgeprägte Vorstellung von „Gesundheit ist machbar“ und „Krankheit ist kontrollierbar“ trifft derzeit auf eine unsichere Erkenntnislage bezüglich der Bedrohung durch das COVID-19-Virus. Die Folge ist eine breite Verunsicherung und viel irrationales Verhalten: von Hamsterkäufen über unbedarftes oder gar provokatives Nichtbefolgen einfachster Verhaltensregeln bis hin zu impliziten oder expliziten Diskriminierungen (etwa vorwurfsvolle Blicke auf asiatische Touristengruppen und gemiedene asiatische Restaurants).

Die Bedrohung durch das COVID-19-Virus wirft viele Fragen zum individuellen und kollektiven Verhalten der Bevölkerung auf. Eine davon ist diejenige nach der Gesundheitskompetenz. Hier geht es um die Fähigkeiten der Bevölkerung,

a. sich gesundheitsrelevante Informationen zu beschaffen und diese zu verstehen - die funktionale Gesundheitskompetenz;

b. sich Gesundheitsinformationen interaktiv anzueignen und sich auszutauschen - die interaktive Gesundheitskompetenz;

c. Gesundheitsinformationen abzuwägen und in den eigenen Kontext zu überführen - die kritische Gesundheitskompetenz.

In der aktuellen Corona-Krise wird nun insbesondere diese kritische Gesundheitskompetenz sehr wichtig: Es geht derzeit nicht nur um einfache Wissensaufnahme, sondern auch und gerade um den produktiven Umgang mit Unsicherheit. Bei Corona ist die Unsicherheit aktuell noch groß: Wissenschaftlich wissen wir vieles noch nicht, die Politik geht schrittweise und teilweise inkohärent vor und die Medien überschwemmen die Bevölkerung mit „facts and fiction“ zu COVID-19.

Die medizinischen Wissenschaften insb. die Virologie und die Epidemiologie brauchen Zeit, um gesicherte Erkenntnisse zu erzielen: Das sollte nicht überraschen und der Bevölkerung klar kommuniziert werden. Die Politik hat es mit hoch komplexen Aufgaben zu tun: Sie muss nicht nur auf die dynamischen Erkenntnisstände der Wissenschaft zeitnah reagieren, sondern auch soziale und ökonomische Bedarfe und Bedürfnisse berücksichtigen. Dies alles dann zu einer ganzheitlichen Politik zusammenfügen - insbesondere in einer liberalen Gesellschaft mit einem föderalen System und immer unter Abwägung von Zwang und Freiheit für den Einzelnen - ist eine mehr als schwere Aufgabe. Die Medien tragen in unterschiedlichem Maß zu Erfolg und Misserfolg der Corona-Strategie bei: Bei ihren Aufgaben der konstruktiven Kritik stoßen sie auf das Problem unsicherer Erkenntnislagen. Ungut wird es, wenn in vielen Medien die Unklarheiten überbetont und Spekulationen verbreitet werden.

Bei realistischer Betrachtung können diese Institutionen (Wissenschaft, Politik, Medien) derzeit noch kein klares Bild zu Corona abgeben. Es kommt so unweigerlich zu einem erheblichen Maß an Verunsicherung in der Bevölkerung. Was dann gebraucht wird, ist kritische Gesundheitskompetenz, einschließlich der Fähigkeit, mit den gegebenen Wissensunsicherheiten umzugehen, Informationen kritisch zu bewerten, die Rolle der Politik sowie auch das eigene Verhalten zu reflektieren.

Um Gesundheitskompetenz und angemessenes Verhalten in der Bevölkerung zu fördern, wird heute häufig auf das Konzept der Selbstverantwortung verwiesen. Dabei zeigt sich die einseitige Betonung der Selbst- oder Eigenverantwortung als problematisch: Für viele steht das Wort Eigenverantwortung in erster Linie für „für sich selbst verantwortlich “ und betont so primär den Eigennutz. In Bedrohungen durch Kontaktinfektionen wie derzeit durch COVID- 
19 ist der Einzelne aber eben nicht nur für sich selbst verantwortlich, sondern gerade auch für die anderen. Für die Mehrzahl der Bevölkerung gilt in der Tat, dass sie nicht zu den Risikogruppen gehören und ihre Verantwortung daher primär den anderen gilt. Das ist schwer zu vermitteln. In Corona-Zeiten muss der Einzelne die schwierige Verbindung von Eigeninteresse und Gemeinwohlverantwortung schaffen.

Gemeinwohlverantwortung ist ein abstrakter und eher schwer zu kommunizierender Begriff. Für die Kommunikation zwischen Wissenschaft und der Bevölkerung, in den Medien und in der Politik erscheint der Begriff der Mitverantwortung wohl angemessener. Er bezeichnet das, was jetzt in der Corona-Krise gebraucht wird, um mit angemessenem Verhalten sich selbst und andere zu schützen und die medizinischen Dienste nicht übermäßig zu beanspruchen. Häufiger von Mitverantwortung zu sprechen, könnte auch helfen, gesellschaftliche und für unser Gesundheitswesen elementare Werte wie Solidarität in die aktuellen Corona-Diskussionen zu integrieren.

Mitverantwortung muss geübt und praktiziert werden. Dafür liefert uns Corona ganz aktuell Anlässe. Das sogenannte „social distancing" und die Schließung öffentlicher Räume kann vielleicht als aktuelles Beispiel dafür dienen, dass der mitverantwortliche Einzelne gefragt ist, um die besten Lösungen zu erreichen: Einen hinreichenden Sicherheitsabstand (1,5-2 Meter) zu den Mitmenschen zu halten, ist eines der effizientesten Mittel, die COVID-19-Übertragungsketten zu durchbrechen. Die Umsetzung der entsprechenden Verhaltensweisen z. B. dadurch zu erreichen, dass man ganze Parkanlagen schließt, basiert auf der Annahme, dass es immer wieder Menschen geben wird, die sich dort treffen und dabei den sicheren Abstand nicht einhalten. Die Wahrscheinlichkeit ist sehr groß, dass diese Menschen sich an einem anderen Ort treffen. Die Alternative wäre wohl, den Park offen zu halten, weiterhin die Möglichkeit zu bieten, Spaziergänge zu machen in einer natürliche Ruhe ausstrahlenden Umgebung, zu erleben, dass man Mitmenschen hat, auch wenn die halt in einer gewissen Distanz bleiben. Evtl. müsste die Anzahl der Besucher begrenzt werden, Parkpersonal könnte angemessenes Verhalten kontrollieren und dort, wo angezeigt, gleich vor Ort zur freundlichen Aufklärung und so zur Gesundheitskompetenz beitragen.

Wenn wir nicht jegliches COVID-19-relevante Verhalten der Menschen regeln können oder wollen, dann braucht es neben strukturellen Vorgaben die (gesundheits-)kompetente Bevölke- rung, die unter Einhaltung der wichtigen Grundregeln (wo immer es geht Abstand halten und oft die Hände waschen) entscheidet, wie sie diese Regeln in ihrem spezifischen Kontext am besten umsetzen kann und so ihrer Mitverantwortung gerecht wird.

In der derzeitigen Krisensituation muss es in erster Linie darum gehen, die Bevölkerung zielführend zu informieren und dazu zu bewegen, mit richtigem Verhalten beizutragen, dass die Ansteckungsketten unterbrochen werden und die aktuelle Krise überwunden wird. Es ist jedoch sehr wahrscheinlich, dass wir zukünftig vermehrt mit ähnlichen Krisen wie die der COVID-19-Infektionen werden umgehen müssen. Hinreichende und besonders auch kritische Gesundheitskompetenz in unserer Bevölkerung wäre ein Teil der Vorbereitung auf Zeiten, die wieder geprägt sein werden von unsicheren Erkenntnisständen und komplexen Maßnahmen. Diese Gesundheitskompetenz muss gelernt werden. Es wird die Aufgabe der Gesellschaft sein, gute Bedingungen dazu zu schaffen, z. B. mit entsprechenden Anpassungen der Lerninhalte in den Schulen. Kritische Gesundheitskompetenz im Sinne des konstruktiv-kritischen Mitwirkens muss auch angewendet werden können. Anwendungsfelder dazu sind z. B. die Mitgestaltung von gesundheitsförderlichen Arbeitsplätzen oder Wohnanlagen. Ebenfalls in diese Richtung gehen heute Projekte im Bereich der sog. „patient reported experiences“, bei denen die Erfahrungen von Patientinnen und Patienten im Gesundheitswesen systematisch erhoben und für Verbesserungen im System genutzt werden können.

Derzeit macht Deutschland neue Erfahrung im Umgang mit einer weitreichenden Bedrohung durch COVID-19. Auch in der aktuellen Krise liegt erhebliches Potenzial für Lernerfahrung. Die politischen Entscheidungsträger müssen z. B. sicherstellen, dass zukünftig bessere infektions- und versorgungsepidemiologischen Daten sehr früh zur Verfügung stehen. Nur so lassen sich Infektionsverläufe valide abbilden und die Erkenntnisse daraus entscheidungsvorbereitend und entscheidungsbegleitenden nutzen.

Zugleich kann und muss aus den sozialen Dynamiken der Corona-Krise gelernt werden. Wie sind die Institutionen und wie die Bürgerinnen und Bürger mit der bedrohlichen Lage umgegangen? Es gilt, diese Erfahrungen der Bevölkerung ernst zu nehmen, systematisch aufzuarbeiten und für die Vorbereitung auf ähnliche Notstände zu nutzen, ganz im Sinne der Förderung von mehr Gesundheitskompetenz und ihrer Nutzung bei aktuellen Gesundheitskrisen. 